\title{
Response to chemotherapy with carboplatin plus albumin-bound paclitaxel in a patient with lymphoepithelioma-like thymic carcinoma: A case report
}

\author{
HIROSHI SHIMA ${ }^{1}$, HIROAKI OZASA ${ }^{1}$, TAKAHIRO TSUJI ${ }^{1}$, HITOMI AJIMIZU ${ }^{1}$, \\ TAKASHI NOMIZO $^{1}$, YOSHITAKA YAGI $^{1}$, YUICHI SAKAMORI ${ }^{1}$, HIROKI NAGAI $^{1}$, \\ SACHIKO MINAMIGUCHI $^{2}$, YOUNG HAK KIM ${ }^{1}$ and MICHIAKI MISHIMA ${ }^{1}$ \\ Departments of ${ }^{1}$ Respiratory Medicine and ${ }^{2}$ Diagnostic Pathology, \\ Kyoto University Graduate School of Medicine, Kyoto 606-8507, Japan
}

Received August 27, 2015; Accepted February 9, 2016

DOI: $10.3892 / \mathrm{mco} .2016 .803$

\begin{abstract}
Thymic carcinoma is a rare neoplasm with a poor outcome due to its aggressive characteristics. For patients who are not operable, radiation therapy and/or palliative chemotherapy are indicated. However, no optimal chemotherapy regimen has been established. The present study reports the case of a 22-year-old man with advanced lymphoepithelioma-like thymic carcinoma refractory to conventional chemotherapy with carboplatin plus solvent-based paclitaxel (sb-PAC) treatment. The patient was subsequently treated with carboplatin plus nanoparticle albumin-bound paclitaxel (nab-PAC). The treatment resulted in a partial response following three cycles of chemotherapy. Since only grade 3 neutropenia, but no other severe adverse effects, was observed, no dose reduction was required. To the best of our knowledge, the current study is the first to present the response to chemotherapy with carboplatin plus nab-PAC in a patient with lymphoepithelioma-like thymic carcinoma. Considering that no standard treatment has been established in thymic carcinoma, nab-PAC may merit further investigation in this rare, but aggressive disease.
\end{abstract}

\section{Introduction}

Thymic tumors, consisting of thymoma and thymic carcinoma, are rare neoplasms that arise in the anterior mediastinum. Thymic carcinomas have malignant cytological features with aggressive invasion of mediastinal organs, resulting in a poor outcome, whereas thymomas are generally considered to be cytologically benign $(1,2)$.

Correspondence to: Dr Takahiro Tsuji, Department of Respiratory Medicine, Kyoto University Graduate School of Medicine, 54 Shogoin-kawaharacho, Sakyo-Ku, Kyoto 606-8507, Japan E-mail: tktsuji@kuhp.kyoto-u.ac.jp

Key words: thymic carcinoma, lymphoepithelioma-like carcinoma, albumin-bound paclitaxel, nab-PAC
Surgery is indicated as the initial treatment for cases in which complete resection is considered feasible. For patients who are not operable, radiation therapy and/or chemotherapy may be indicated for symptom control and potentially prolonged survival rates (3). Owing to the rarity of thymic carcinoma, however, a standard chemotherapy regimen remains to be established.

Nanoparticle albumin-bound paclitaxel (nab-PAC), an albumin-bound, $130 \mathrm{~nm}$ particle form of paclitaxel, was developed to avoid Cremophor/ethanol-associated toxicities for solvent-based paclitaxel (sb-PAC), and to exploit albumin receptor-mediated endothelial transport (4). Promising activity was demonstrated in patients with non-small cell lung cancer (NSCLC), breast cancer and gastric cancer (5-7). In the present study, a case of lymphoepithelioma-like thymic carcinoma is described, refractory to carboplatin plus sb-PAC (CP) treatment, but which responded to subsequent treatment with carboplatin plus nab-PAC.

\section{Case report}

A 22-year-old man presented with progressive chest pain, dyspnea and body weight loss. Contrast-enhanced computed tomography (CT) using an Aquillion ${ }^{\mathrm{TM}}$ One CT scanner (Toshiba, Tokyo, Japan) revealed a 20x10 cm irregular tumor in the right thorax with pleural dissemination and invasion to the brachiocephalic trunk, right pulmonary artery, trachea and epicardium (Fig. 1).

Fluorodeoxyglucose (FDG) positron-emission CT (Discovery IQ; GE Healthcare, Buckinghamshire, UK) demonstrated marked FDG accumulation in the tumor. CT-guided needle biopsy of the tumor was performed. The tissue was fixed in $10 \%$ neutral-buffered formalin, and embedded in paraffin. A $3 \mu \mathrm{m}$-thick section of the paraffin block was stained with hematoxylin and eosin, and used for immunohistochemistry. Histologically, the tumor was composed of nests and sheets of malignant cells with the infiltration of lymphocytes (Fig. 2A). The tumor cells exhibited a high nuclear/cytoplasmic ratio and large vesicular nuclei with distinct nucleoli. Immunohistochemical staining was performed on a Ventana 
A

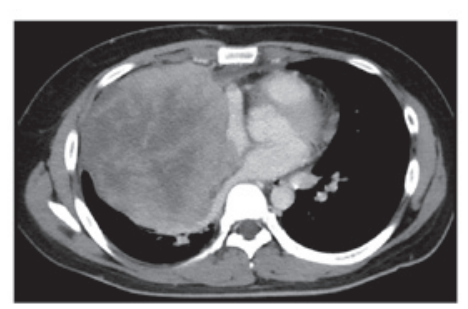

B

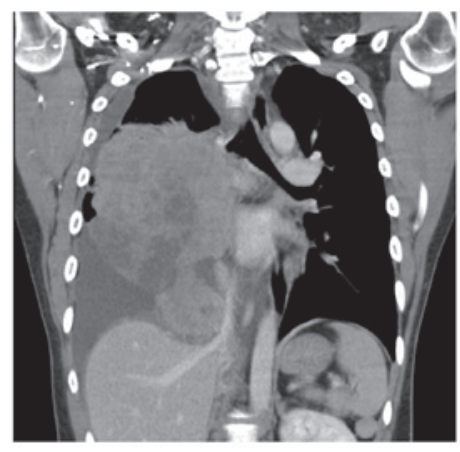

Figure 1. Imaging findings of chest CT at diagnosis. (A) A contrast-enhanced CT image, showing a 20x10 $\mathrm{cm}$ tumor. (B) A coronal section image of the CT, revealing pleural fluid and the mass invading mediastinal organs: The brachiocephalic trunk, right pulmonary artery, trachea and epicardium. CT, computed tomography.

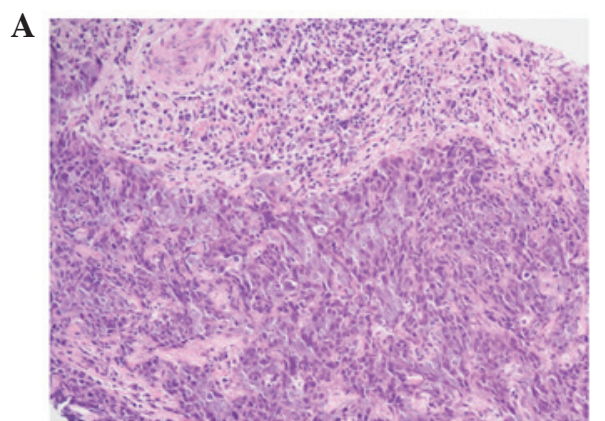

$\mathbf{B}$

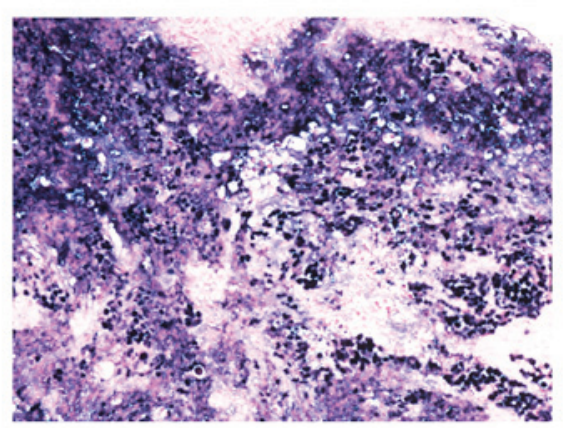

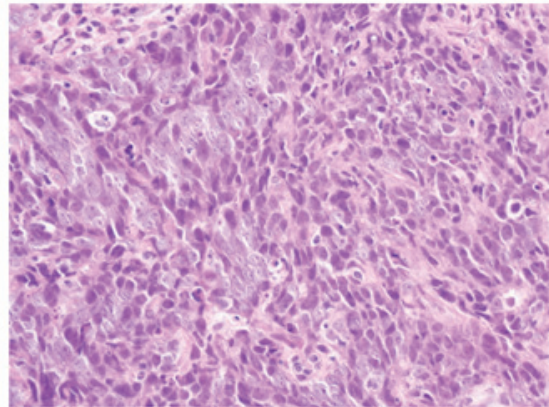

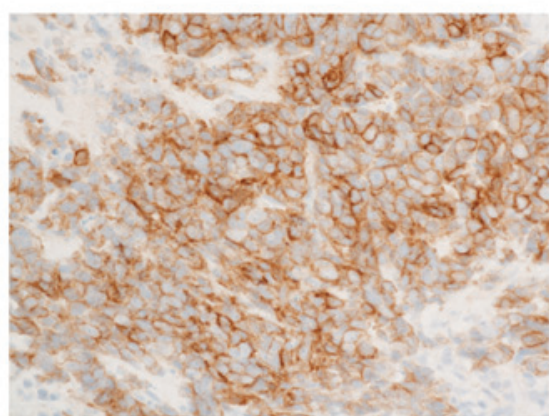

Figure 2. Histopathological findings of the tumor obtained by CT-guided needle biopsy. (A) Low-power (left) and high-power (right) images of a hematoxylin and eosin-stained section, showing the tumor consisting of sheets and nests of carcinoma cells with indistinct cell borders accompanied by abundant lymphocytes. (B) Immunohistochemical analysis, showing positivity for Epstein-Barr virus-encoded small RNA (left) and CD117 (right). CT, computed tomography.

BenchMark URTLA instrument (Ventana Medical Systems, Inc., Tucson, AZ, USA). CAM5.2 (clone cytokeratin 8, 1:500, B.D. 349205), cluster of differentiation 5 (CD5; clone 4C7, 1:50, Leika NCL-CD5-4C7), CD117 (polyclonal, 1:200, Dako A4502) and EBER [Epstein-Barr virus (EBV)-encoded small RNA] in situ hybridization (ISH; Roche 518-102524) were selected as diagnostic markers. Immunohistochemical staining revealed that the tumor cells were positive for cytokeratin CAM5.2, CD117 and the EBV-encoded small RNA, but were negative for CD5 (Fig. 2B). On the basis of these findings, the patient was diagnosed with lymphoepithelioma-like thymic carcinoma, stage IVa, in accordance with the Masaoka-Koga staging system (8).

As the initial therapy, palliative radiotherapy (45 Gy) for the mediastinal area in combination with weekly carboplatin [dose calculation: Area under curve (AUC) 2] plus sb-PAC $\left(40 \mathrm{mg} / \mathrm{m}^{2}\right)$ was started to palliate the airway obstruction and dyspnea. When the palliative radiotherapy was completed, despite the reduction in tumor size, liver invasion was subsequently demonstrated using FDG positron emission CT (Fig. 3A). Subsequently, chemotherapy with carboplatin (AUC 6, day 1) and nab-PAC (100 mg/m $\mathrm{m}^{2}$, days 1,8 and 15$)$ every 3 weeks was started. Since only grade 3 neutropenia, but no other severe adverse effects, was observed, no dose reduction was required. Objective tumor size reduction (partial response) was obtained following three cycles of chemotherapy (Fig. 3B).

\section{Discussion}

Thymic carcinoma is a rare neoplasm, with a poor outcome due to its aggressive characteristics. For patients who are not operable, radiation therapy and/or palliative chemotherapy are indicated (1). Since a standard chemotherapy regimen for thymic carcinoma remains to be established, the present case report implies that carboplatin plus nab-PAC is a promising therapy for patients with thymic carcinoma. 

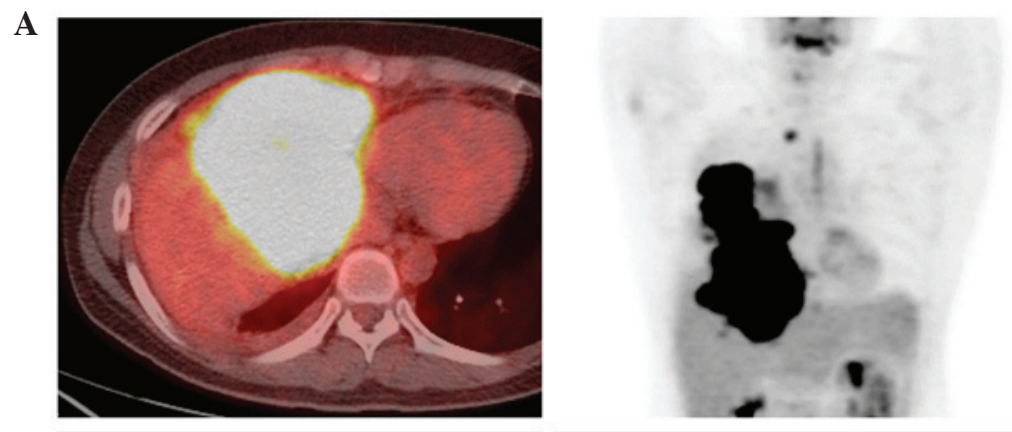

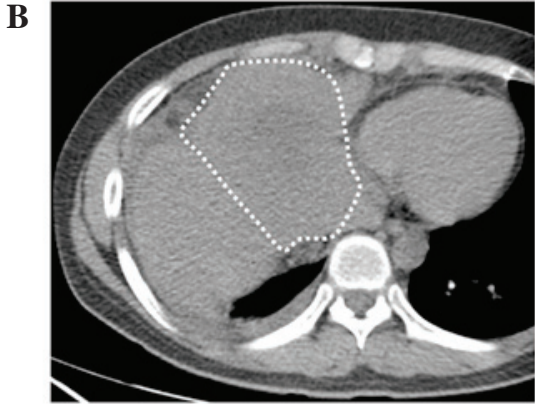

Before

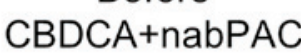

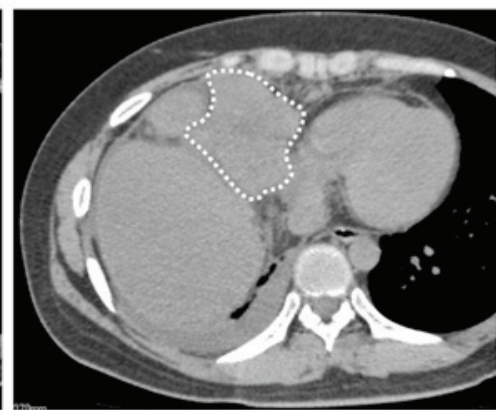

After 3 cycles of CBDCA+nabPAC

Figure 3. Response to chemotherapy with CBDCA with nab-PAC. (A) Fluorodeoxyglucose positron emission CT images showing liver invasion during conventional chemotherapy with CBDCA plus solvent-based paclitaxel. (B) Plain CT images prior to chemotherapy with CBDCA plus nab-PAC (left); following three cycles of chemotherapy (right), the reduction in the tumor size is shown. White dotted lines show the margins of the tumor. CBDCA, carboplatin; CT, computed tomography; nab-PAC, nanoparticle albumin-bound paclitaxel.

In the National Comprehensive Cancer Network guidelines for thymoma and thymic carcinoma, $\mathrm{CP}$ is recommended as the first-line chemotherapy in patients with advanced-stage thymic carcinoma (3). Two phase II studies by Lemma et al (9) and Hirai et al (10) revealed that patients with advanced-stage thymic carcinoma had a favorable response to $\mathrm{CP}$ (overall response rate of 22 and $36 \%$, respectively) compared with the response in other studies (11-18). There have been no prospective large-scale trials addressing the optimal chemotherapy regimen for patients with thymic carcinoma, due to the rarity of this disease.

$\mathrm{CP}$ regimens occasionally cause severe sensory neuropathy, resulting in dose reduction and/or treatment termination. In the study of Lemma et al (9), grade 3 sensory neuropathy was observed in $13.3 \%$ [and the rate of discontinuation of treatment for toxicity was considerably higher $(\sim 21 \%)]$ of the patients with either thymoma or thymic carcinoma receiving the $\mathrm{CP}$ regimen (9).

Nab-PAC, the $130 \mathrm{~nm}$ albumin-bound paclitaxel formulation, is a promising novel agent with lower toxicity and higher accumulation in tumors compared with sb-PAC. In a preclinical in vivo study, increased antitumor activity of nab-PAC was reported, with a $33 \%$ higher paclitaxel concentration in tumors compared with sb-PAC (4). A phase III trial for patients with advanced NSCLC revealed that the administration of carboplatin plus nab-PAC as a first-line therapy resulted in a significantly lower rate of sensory neuropathy, $26 \%$ greater dose intensity, and a higher objective response rate ( 33 vs. $25 \% ; \mathrm{P}=0.005$ ) compared with CP (5). In the current case study, weekly CP was administered in combination with palliative radiotherapy due to the safe profile of $\mathrm{CP}$ when administered with concurrent radiotherapy.
Notably, the tumor responded to subsequent chemotherapy with carboplatin and nab-PAC, despite progression during the treatment with weekly CP. A high accumulation of nab-PAC in the tumor and high dose intensity may have contributed to the response.

According to the World Health Organization classification, there are 13 subtypes of thymic carcinoma: $60-70 \%$ of all thymic carcinomas are subtypes of squamous cell carcinoma and lymphoepithelioma-like carcinoma. The lymphoepithelioma-like carcinoma is a classically common, although now rare, subtype (1.0-8.2\%), with poor prognosis, being associated with Epstein-Barr virus infection (19-25). A histological difference appears to be associated with the response to nab-PAC: A subset analysis of a phase III study in patients with NSCLC revealed that squamous cell carcinoma responded markedly well to carboplatin plus nab-PAC (5). In thymic carcinoma, two cases receiving carboplatin plus nab-PAC and exhibiting a favorable response were previously reported, and notably, the two cases were presented as squamous cell carcinomas $(26,27)$. The current case study describes, to the best of our knowledge, the first patient with thymic lymphoepithelioma-like subtype who responded to carboplatin plus nab-PAC. A response to nab-PAC, in association with the histological subtype, in thymic carcinoma should be discussed in the future.

In conclusion, a case of thymic carcinoma with lymphoepithelioma-like histology that responded to treatment with carboplatin plus nab-PAC has been presented in the current study. Considering that a standard treatment has yet to be established in patients with thymic carcinoma, nab-PAC may merit further investigation in this rare, but aggressive disease. 


\section{References}

1. Falkson CB, Bezjak A, Darling G, Gregg R, Malthaner R, Maziak DE, Yu E, Smith CA, McNair S, et al: The management of thymoma: A systematic review and practice guideline. J Thorac Oncol 4: 911-919, 2009.

2. Eng TY, Fuller CD, Jagirdar J, Bains Y and Thomas CR Jr: Thymic carcinoma: State of the art review. Int J Radiat Oncol Biol Phys 59: 654-664, 2004

3. NCCN guidelines on the treatment of thymomas and thymic carsinomas. (Version 1.2014: http ://www.nccn.org/professionals/physician_gls/pdf/thymic.pdf. Accessed July 3, 2015.

4. Desai N, Trieu V, Yao Z, Louie L, Ci S, Yang A, Tao C, De T, Beals B, Dykes D, et al: Increased antitumor activity, intratumor paclitaxel concentrations and endothelial cell transport of cremophor-free, albumin-bound paclitaxel, ABI-007, compared with cremophor-based paclitaxel. Clin Cancer Res 12: 1317-1324, 2006.

5. Socinski MA, Bondarenko I, Karaseva NA, Makhson AM, Vynnychenko I, Okamoto I, Hon JK, Hirsh V, Bhar P,Zhang H, et al: Weekly nab-paclitaxel in combination with carboplatin versus solvent-based paclitaxel plus carboplatin as first-line therapy in patients with advanced non-small-cell lung cancer: Final results of a phase III trial. J Clin Oncol 30: 2055-2062, 2012.

6. Gradishar WJ, Tjulandin S, Davidson N, Shaw H, Desai N, Bhar P, Hawkins M and O'Shaughnessy J: Phase III trial of nanoparticle albumin-bound paclitaxel compared with polyethylated castor oil-based paclitaxel in women with breast cancer. J Clin Oncol 23: 7794-7803, 2005.

7. Koizumi W, Morita S and Sakata Y: A randomized Phase III trial of weekly or 3-weekly doses of nab-paclitaxel versus weekly doses of Cremophor-based paclitaxel in patients with previously treated advanced gastric cancer (ABSOLUTE Trial). Jpn J Clin Oncol 45: 303-306, 2015

8. Masaoka A, Monden Y, Nakahara K and Tanioka T: Follow-up study of thymomas with special reference to their clinical stages. Cancer 48: 2485-2492, 1981.

9. Lemma GL, Lee JW, Aisner SC, Langer CJ, Tester WJ, Johnson DH and Loehrer PJ Sr: Phase II study of carboplatin and paclitaxel in advanced thymoma and thymic carcinoma. J Clin Oncol 29 : 2060-2065, 2011

10. Hirai F, Yamanaka T, Taguchi K, Daga H, Ono A, Tanaka K, Kogure Y, Shimizu J, Kimura T, Fukuoka J, et al: A multicenter phase II study of carboplatin and paclitaxel for advanced thymic carcinoma: WJOG4207L. Ann Oncol 26: 363-368, 2015.

11. Koizumi T, Takabayashi Y, Yamagishi S, Tsushima K, Takamizawa A, Tsukadaira A, Yamamoto H, Yamazaki Y, Yamaguchi S, Fujimoto K, et al: Chemotherapy for advanced thymic carcinoma: Clinical response to cisplatin, doxorubicin, vincristine and cyclophosphamide (ADOC chemotherapy). Am J Clin Oncol 25: 266-268, 2002

12. Kanda S, Koizumi T, Komatsu Y, Yoshikawa S, Okada M, Hatayama O, Yasuo M, Tsushima K, Urushihata K, Kubo K, et al: Second-line chemotherapy of platinum compound plus CPT-11 following ADOC chemotherapy in advanced thymic carcinoma: Analysis of seven cases. Anticancer Res 27: 3005-3008, 2007.

13. Komatsu Y, Koizumi T, Tanabe T, Hatayama O, Yasuo M Okada M, Yamamoto H, Kubo K, Sasabayashi M and Tsunoda T: Salvage chemotherapy with carboplatin and paclitaxel for cisplatin-resistant thymic carcinoma-three cases. Anticancer Res 26: 4851-4855, 2006.
14. Weide LG, Ulbright TM, Loehrer PJ Sr and Williams SD: Thymic carcinoma. A distinct clinical entity responsive to chemotherapy. Cancer 71: 1219-1223, 1993.

15. Yoh K, Goto K, Ishii G, Niho S, Ohmatsu H, Kubota K, Kakinuma R, Nagai K, Suga M and Nishiwaki Y: Weekly chemotherapy with cisplatin, vincristine, doxorubicin and etoposide is an effective treatment for advanced thymic carcinoma. Cancer 98: 926-931, 2003

16. Lucchi M, Mussi A, Ambrogi M, Gunfiotti A, Fontanini G, Basolo F and Angeletti CA: Thymic carcinoma: A report of 13 cases. Eur J Surg Oncol 27: 636-640, 2001.

17. Igawa S, Murakami H, Takahashi T, Nakamura Y, Tsuya A, Naito T, Kaira K, Ono A, Shukuya T, Tamiya A, et al: Efficacy of chemotherapy with carboplatin and paclitaxel for unresectable thymic carcinoma. Lung Cancer 67: 194-197, 2010.

18. Maruyama R, Suemitsu R, Okamoto T, Kojo M, Aoki Y, Wataya H, Eguchi T, Nishiyama K, Seto T and Ichinose Y: Persistent and aggressive treatment for thymic carcinoma. Results of a single-institute experience with 25 patients. Oncology 70: 325-329, 2006.

19. Leyvraz S, Henle W, Chahinian AP, Perlmann C, Klein G, Gordon RE, Rosenblum M and Holland JF: Association of Epstein-Barr virus with thymic carcinoma. N Engl J Med 312: 1296-1299, 1985.

20. Schirosi L, Nannini N, Nicoli D, Cavazza A, Valli R, Buti S, Garagnani L, Sartori G, Calabrese F, Marchetti A, et al: Activating c-KIT mutations in a subset of thymic carcinoma and response to different c-KIT inhibitors. Ann Oncol 23: 2409-2414, 2012.

21. Kondo K and Monden Y: Therapy for thymic epithelial tumors: A clinical study of 1,320 patients from Japan. Ann Thorac Surg 76: 878-884; discussion 884-885, 2003.

22. Okuma Y, Hosomi Y, Takahashi S, Maeda Y, Okamura T and Hishima T: Response to cytotoxic chemotherapy in patients previously treated with palliative-intent chemotherapy for advanced thymic carcinoma. Clin Lung Cancer 16: 221-227, 2015.

23. Li W, Miao Z, Liu X, Zhang Q, Sun L, Li P, Liu W and Zhang L: Thymic carcinoma patients with myasthenia gravis exhibit better prognoses. Int J Clin Oncol, 2015 Jul 3 [Epub ahead of print].

24. Filosso PL, Guerrera F, Rendina AE, Bora G, Ruffini E, Novero D, Ruco L, Vitolo D, Anile M, Ibrahim M, et al: Outcome of surgically resected thymic carcinoma: A multicenter experience. Lung Cancer 83: 205-210, 2014.

25. Wang S, Wang Z, Liu X, Wang D and Liu F: Prognostic factors of patients with thymic carcinoma after surgery: A retrospective analysis of 58 cases. World J Surg 38: 2032-2038, 2014.

26. Makimoto G, Fujiwara K, Watanabe H, Kameyama N, Matsushita M, Rai K, Sato K, Yonei T, Sato T and Shibayama T: nab-Paclitaxel in combination with carboplatin for a previously treated thymic carcinoma. Case Rep Oncol 7: 14-17, 2014.

27. Zhan P, Xie H and Yu LK: Response to nab-paclitaxel and nedaplatin in a heavily-metastatic thymic carcinoma: A case report. Oncol Lett 9: 1715-1718, 2015. 